\title{
Philippe Zawieja, Franck Guarnieri (dir.), Dictionnaire des risques psychosociaux
}

Paris, Seuil, 2014

\section{Rémy Ponge}

\section{OpenEdition}

\section{Journals}

Édition électronique

URL : http://journals.openedition.org/travailemploi/6512

DOI : 10.4000/travailemploi.6512

ISSN : 1775-416X

Éditeur

DARES - Ministère du Travail

Édition imprimée

Date de publication : 1 octobre 2014

Pagination : $67-70$

ISSN : 0224-4365

\section{Référence électronique}

Rémy Ponge, "Philippe Zawieja, Franck Guarnieri (dir.), Dictionnaire des risques psychosociaux », Travail et Emploi [En ligne], 140 | octobre-décembre 2014, mis en ligne le 01 octobre 2014, consulté le 10 décembre 2020. URL : http://journals.openedition.org/travailemploi/6512 ; DOI : https://doi.org/ 10.4000/travailemploi.6512

Ce document a été généré automatiquement le 10 décembre 2020.

(c) Direction de l'animation de la recherche, des études et des statistiques (Dares) 


\section{Philippe Zawieja, Franck Guarnieri (dir.), Dictionnaire des risques psychosociaux}

Paris, Seuil, 2014

Rémy Ponge

\section{RÉFÉRENCE}

Philippe Zawieja, Franck Guarnieri (dir.), Dictionnaire des risques psychosociaux, Paris, Seuil, 2014, $882 \mathrm{p}$.

Dans tout domaine de connaissances, il est des notions dont le succès est au moins égal aux controverses et aux critiques qu'elles ont suscitées. C'est sans aucun doute le cas des risques psychosociaux (RPS), notion qui a connu un important retentissement ces dix dernières années en France mais dont la définition, le contenu, le périmètre et les usages provoquent d'intenses débats. C'est la volonté d'apporter de la clarté à ce qu'ils appellent cette «cacophonie psychosociale » qui a conduit Philippe Zawieja et Franck Guarnieri, respectivement chercheur associé et directeur de recherche au Centre de recherches sur les risques et les crises de l'École des mines de Paris, à coordonner la rédaction du Dictionnaire des risques psychosociaux. Curieux objet a priori qu'un dictionnaire, recueil de définitions stabilisées, pour un sujet aussi controversé que celui des RPS. Mais qu'on ne s'y méprenne pas, l'intention des auteurs n'est pas d'énoncer à travers cet ouvrage une énième définition des risques psychosociaux. Elle est plutôt de rassembler et de mettre à plat la masse de connaissances hétérogènes produites sur le sujet. Illustration de cette volonté de laisser ouvert l'espace des débats, on ne trouvera pas d'entrée RPS parmi les 314 contributions qui constituent cet imposant ouvrage de presque 900 pages dont il est bien entendu impossible de restituer ici l'hétérogénéité et la richesse du contenu. Notre ambition sera plutôt de proposer des points de réflexion 
sur l'apport de ce dictionnaire qui, comme tout recueil de ce type, a davantage vocation à être régulièrement consulté que lu d'une traite.

\section{Un contenu essentiellement académique aux approches disciplinaires variées}

2252 auteurs, 150 hommes et 102 femmes, ont participé à la rédaction du Dictionnaire des risques psychosociaux. Cet ouvrage est principalement l'œuvre de chercheurs puisque 147 universitaires, 18 chercheurs au Conservatoire national des arts et métiers (Cnam), 17 en écoles de commerce et de management, 9 membres du CNRS et 7 de l'École des mines y ont contribué, auxquels s'ajoute une trentaine de chercheurs rattachés à diverses institutions publiques ou privées. Si l'ouvrage fait place à des auteurs aux appartenances institutionnelles variées et issus de différentes disciplines, la sociologie et la psychologie au sens large, incluant notamment la psychologie du travail (24), et la psychologie sociale (11) arrivent largement en tête avec respectivement 65 et 61 auteurs. Viennent ensuite la gestion et le management (27), le droit (16), l'ergonomie (10), la médecine (6), l'épidémiologie (5) et l'économie (3). D'autres disciplines sont également présentes, comme les sciences de l'information et de la communication (2), l'ethnologie (2), la philosophie (2) ou encore l'histoire (1). Il faut par ailleurs saluer la place faite à de jeunes auteurs, notamment aux doctorants (7).

3 La présence de différentes disciplines souligne l'important intérêt académique que soulève la thématique des risques psychosociaux et, plus largement, de la santé au travail, comme l'illustrent par ailleurs les programmes de recherches soutenus par l'Agence nationale de la recherche ${ }^{1}$, les enquêtes conduites par la Direction de l'animation de la recherche, des études et des statistiques (Dares) comme Sumer (Surveillance médicale des expositions aux risques professionnels) ou SIP (Santé et itinéraire professionnel), ou encore la création du Groupe d'études sur la souffrance et le travail (Gestes), réseau constitué en 2011 et financé par la région Île-de-France, qui rassemble plus de 200 chercheurs issus de différentes disciplines.

4 La présence d'approches s'appuyant sur la psychologie et ses divers courants parmi les contributions de l'ouvrage reflète sans doute l'intérêt ancien de cette discipline pour les troubles de la santé mentale en lien avec le travail. Quant aux sociologues, leur attention a depuis longtemps été retenue par les questions relatives au travail, à son organisation, à ses conditions concrètes de réalisation ainsi que par l'analyse des évolutions des organisations. C'est ce que montrent les entrées «organisations du travail», «conditions de travail», «intensification" ou encore "chômage » ou «travail précaire » qui leur sont confiées. Le lecteur désireux de se familiariser avec les multiples disciplines et la spécificité des démarches (méthodologiques, théoriques) et des concepts qu'elles proposent sur les questions de santé mentale en trouvera de riches et synthétiques présentations comme celles, entre autres, de "l'ergonomie », de la «psychodynamique du travail», de la «psychopathologie du travail», de la « clinique de l'activité » ou encore de « l'épidémiologie ».

5 Si ce dictionnaire accorde une place centrale aux acteurs de la recherche, il donne également la parole aux praticiens qui constituent $15 \%$ des auteurs et sont majoritairement issus du champ de la santé, de la psychologie ou du conseil: consultants (12), psychiatres (8), médecins du travail et de prévention (5), psychologues cliniciens ou du travail (4), médecins (2) et psychanalystes. On trouve aussi des acteurs 
que l'on entend généralement moins sur ces questions comme les experts judiciaires (3) ou les assistantes sociales (1). Bien qu'un tiers des contributeurs de l'ouvrage viennent de Paris (80), on en trouve aussi venus de toute la France (113) et de pays étrangers (41), très largement francophones (Canada, Belgique, Suisse). Si l'ouvrage s'adresse donc à un public francophone, voire français, tant au niveau de ses contributeurs que du contenu - la plupart des entrées concernent la France -, il ne s'y cantonne pas pour autant et ouvre des perspectives internationales à travers des entrées spécifiques (Chine, Japon, karoshi ${ }^{2}$, etc.) ou présentant des études menées dans d'autres pays (ÉtatsUnis, Angleterre, Suède, Norvège, etc.). Chacune d'elles est par ailleurs traduite en anglais, et s'achève le plus souvent par une courte bibliographie contenant des références en anglais, ce qui facilitera, pour le lecteur curieux, l'exploration de la littérature anglo-saxonne. Un système de renvois, une brève présentation de chaque contributeur ainsi qu'une liste des entrées et un index permettent une navigation efficace dans cet ouvrage néanmoins tellement foisonnant qu'il peut être parfois déroutant.

\section{Des entrées extrêmement diversifiées}

6 En effet, si l'on se penche plus en avant sur le contenu des contributions, on est frappé par la grande diversité des entrées tant en termes de taille, certaines se concentrant sur moins d'une page et d'autres occupant plus de cinq pages, que de contenu, certaines proposant ainsi d'audacieuses synthèses de phénomènes très généraux (" néolibéralisme », « hypermodernisation », « accélération », etc.) tandis que d'autres s'attachent à la description d'objets plus circonscrits comme par exemple le cadre légal et les usages possibles du certificat médical. Sans prétendre à l'exhaustivité, le lecteur peut toutefois s'essayer à quelques regroupements afin d'y voir plus clair dans le contenu de cet ouvrage.

\section{... Par le cadre juridique et les acteurs}

7 Ainsi, une série d'entrées détaille les multiples éléments du cadre juridique de la santé au travail en matière de risques psychosociaux («droit à la protection de la santé mentale au travail», "obligation de sécurité de résultat de l'employeur", "prévention», «réparation», « risques professionnels», " accident du travail», «maladie professionnelle », etc.). Plusieurs de ces contributions présentent un intérêt particulier pour le praticien dans la mesure où elles détaillent dans quels contextes et de quelles manières mobiliser toute une série d'outils juridiques pour faire face aux risques psychosociaux ("droit d'alerte», «droit de retrait», «document unique d'évaluation des risques professionnels», etc.). Les nombreux acteurs de la santé au travail font bien entendu l'objet d'entrées spécifiques: acteurs de l'entreprise ("institutions représentatives du personnel », " $\mathrm{CHSCT}^{3}$ », " médecin du travail», " syndicats ", " managers ", etc.), acteurs externes (" préventeurs », " intervenants en prévention des risques professionnels", «inspection du travail ») mais aussi acteurs institutionnels ("Anses ${ }^{4} »$, «Anact $\left.{ }^{5} », ~ « \mathrm{INRS}^{6} »\right)$. Toutes ces entrées permettent de mieux cerner les spécificités de chacun de ces acteurs ainsi que le rôle qu'ils peuvent jouer en matière de RPS. 


\section{... Par les résultats de recherches empiriques sur certains métiers}

8 Un ensemble d'entrées livre les multiples résultats de recherches empiriques sur certains métiers. On y retrouve des cas emblématiques des travaux sur les risques psychosociaux tels que ceux sur les "cadres ", les « infirmières ", les " enseignants ", les salariés des "centres d'appels", les "agriculteurs", les "policiers", mais aussi ceux sur des travailleurs plus rarement associés aux problématiques de risques psychosociaux comme les « éboueurs » ou les « vétérinaires ». On y apprend ainsi qu'en Grande-Bretagne, plus de $20 \%$ des vétérinaires souffrent de dépression et que la mortalité par suicide (cinq ou six par an) y est quatre fois supérieure à celle de la "population générale et deux fois supérieure à celle des professions de santé ». Seraient en cause l'isolement et la rareté des structures permettant l'échange entre professionnels, la charge émotionnelle liée à l'accompagnement de la souffrance des animaux et de la tristesse de leur propriétaire, mais aussi la surcharge de travail et les difficultés entrainées par la gestion comptable des cabinets à laquelle les vétérinaires n'ont pas été formés. Cette galerie de problématiques de santé mentale propres à différents métiers est enrichie par un ensemble de contributions plus générales qui achèvent de souligner la variété des situations de travail et des métiers concernés ( " métiers de la forêt », « open space ", " vente », " grande distribution », etc.).

\section{... Par les pathologies associées}

9 Signe supplémentaire de la diversité des situations couvertes par la notion de RPS, l'ouvrage s'ouvre sur «absentéisme » et se clôt sur « workaholism ». Le lecteur désireux d'en apprendre plus sur les pathologies et les formes de souffrance associées aux risques psychosociaux pourra d'ailleurs trouver de quoi satisfaire son appétit. À côté des pathologies bien identifiées comme relevant des RPS telles que la « dépression », les différentes formes de harcèlement, "l'épuisement professionnel» ou encore le "suicide», on en découvrira de nombreuses autres: "solitude», " ennui organisationnel », « anxiété», «mépris», « humiliation», "placard», etc. Ce foisonnement d'entrées renvoyant à des formes variées de souffrance au travail souligne l'approche extensive de la notion de risques psychosociaux retenue par les auteurs, qui semblent avoir eu le souci de donner au lecteur l'éclairage le plus exhaustif possible.

Les auteurs ont par ailleurs souhaité livrer des éléments permettant d'appréhender les liens multiples et mouvants entre situations hors travail et risques psychosociaux. Outre la classique articulation «vie familiale/vie professionnelle», l'entrée «migration », qui présente les multiples causes de souffrance auxquelles sont exposés les migrants tout au long de leur parcours et de leur expérience migratoire, invite le lecteur à prêter attention à ce qui se joue en dehors de la sphère du travail. Cette entrée a le mérite d'attirer le regard sur des situations encore peu étudiées et de s'interroger sur la spécificité de l'expérience de travail de certaines populations, ici les migrants en situation irrégulière.

\section{... Par des points de vue contrastés sur l'action en matière de santé au travail}

11 F. Guarnieri et P. Zawieja, fidèles à leur ambition initiale, n'ont par ailleurs pas hésité à multiplier les éclairages sur la notion de RPS et les termes qu'elle recouvre - comme pour le stress qui compte plus de cinq entrées différentes - ainsi que les contributions 
défendant des points de vue contrastés sur ce qu'est le travail, la manière dont il convient de l'analyser et d'agir pour assurer la santé mentale des salariés. L'entrée "facteurs psychosociaux » nous incite ainsi à réfléchir à une manière de les quantifier. Selon ses auteurs, en s'appuyant sur une série de mesures objectives prenant en compte un ensemble de facteurs, il est possible d'établir un seuil à partir duquel tel facteur devient, en règle générale, un risque pour les salariés. On peut ainsi mesurer le soutien social apporté par un gestionnaire à ses employés en évaluant son ratio de supervision, la distance physique qui le sépare de ses subordonnés, le temps qu'il passe en réunion, en transport ou en formation, etc. Bien sûr, la question de savoir si cette évaluation " objective" rejoint l'évaluation "subjective» faite par les salariés demeure. Mais, selon les auteurs, ce type de mesures pourrait servir à l'établissement de valeurs limites d'exposition qui soutiendraient la mise en place de stratégies de prévention des risques. D'autres entrées plaident davantage pour une intervention centrée sur l'individu; c'est le cas de l'entrée «mindfulness ». Cet état de " pleine conscience » axé sur le présent et caractérisé par une absence de jugement permet au travailleur de recueillir et d'accepter telle quelle chaque pensée qui «survient dans le champ de sa conscience ». Le travailleur mindful serait ainsi mieux à même d'accueillir les «émotions perturbatrices » engendrées par son activité, de renouveler ses "schémas de pensée ", de gérer son stress et de libérer sa créativité. L'objectif d'une intervention visant à améliorer la santé au travail est alors, via un "protocole d'entraînement " (huit séances hebdomadaires complétées par des exercices quotidiens), d'amener chaque travailleur à se libérer "des pensées et des jugements inconscients » qui le conduisent à voir de nombreux événements comme des problèmes ou des difficultés. À rebours de ces deux approches, l'entrée " clinique de l'activité » met en garde contre ce qu'elle appréhende comme une requalification de «situations de travail saturées de problèmes » en "fragilité individuelle ». Ici, il ne s'agit pas de chercher à "soigner les individus » en les aidant à développer des qualités individuelles leur permettant, par exemple, de mieux gérer leur stress. Il ne faut pas non plus s'efforcer de mesurer de la manière la plus "objective" possible les facteurs de risques auxquels peuvent être exposés les travailleurs, ce qui tendrait à en faire des acteurs passifs de l'activité et à les éloigner du débat sur les critères du «bon travail», point nodal de la santé au travail. Car, selon la " clinique de l'activité », c'est précisément le déni du " conflit ordinaire » sur le travail bien fait qui constitue le principal risque psychosocial. Le travail de qualité est par nature discutable et la confrontation des salariés au réel fait surgir de multiples imprévus qui suscitent des dilemmes et nourrissent des conflits. L'enjeu est alors d'amener les travailleurs à retrouver des capacités d'action et à les faire débattre entre eux et avec leur hiérarchie des critères du travail de qualité.

\section{Les RPS, une notion au croisement de la science et du politique?}

12 À la lecture des multiples contributions de ce dictionnaire on est finalement conduit à s'interroger sur ce qui fonde, en définitive, la spécificité de la notion de risques psychosociaux tant elle semble parfois se diluer dans un large spectre de notions hétérogènes. À cette question, l'entrée consacrée à «l'épistémologie des risques psychosociaux » apportera un éclairage utile. En effet, l'auteur y rappelle que la notion a été construite à partir de l'épidémiologie sociale suédoise et des travaux précurseurs réalisés dans les années 1970 par Lennart Levi ou encore Bertil Gardell sur la notion de « contrôle » du travailleur sur son propre travail. Cette recherche suédoise, qui se veut 
clairement au service d'une "politique sociale réformiste", influencera la législation sur le travail en Suède. L'importation de la notion de risque psychosocial, contraction des termes «facteur psychosocial » et «facteur de risque », dans le champ intellectuel français à partir des années 1990 puis 2000, est, quant à elle, liée à la mise en place d'une politique de santé et sécurité au travail européenne et à la transcription dans le droit français de la directive cadre de 1989, qui instaure notamment pour l'employeur l'obligation de garantir la santé et la sécurité des travailleurs. Plusieurs instances internationales et européennes contribueront également à cette importation, comme la Commission européenne et l'Organisation mondiale de la santé, où Lennart Levi est d'ailleurs un acteur influent, mais aussi le Bureau international du travail et l'Agence européenne pour la sécurité et la santé au travail. Cette entrée nous rappelle ainsi que cette notion controversée de risques psychosociaux s'inscrit au croisement de la science et de la politique.

Une fois arrivés à la fin de ce dictionnaire, y voyons-nous donc plus clair sur cette "cacophonie psychosociale» dont parlaient les auteurs? On pourra regretter qu'un nombre restreint seulement de contributions apporte un recul historique sur cette thématique et sur les nombreux termes mobilisés, alors que le thème de la santé au travail est aussi travaillé par de nombreux chercheurs historiens ou sociohistoriens ${ }^{7}$. Face à la somme de savoirs produits sur le sujet, l'historicisation plus systématique des concepts et un retour sur leur contexte et leurs conditions de production apporteraient un éclairage supplémentaire précieux. Toutefois, tel n'est pas l'objet de ce dictionnaire qui constituera un support utile pour appréhender la multiplicité et la grande variété des connaissances produites sur le thème des risques psychosociaux.

\section{NOTES}

1. Programme santé-environnement et santé-travail (2005-2007) et programme environnementsanté-travail (2010-2014).

2. Mort par excès de travail.

3. Comité d'hygiène, de sécurité et des conditions de travail.

4. Agence nationale de sécurité sanitaire de l'alimentation, de l'environnement et du travail.

5. Agence nationale pour l'amélioration des conditions de travail.

6. Institut national de recherche et de sécurité.

7. Par exemple, et de façon non exhaustive, Jean-Claude Devinck, Éric Geerkens, Nicolas Hatzfeld, Pascal Marichalar, Catherine Omnès, Laure Pitti, Judith Rainhorn, Paul-André Rosental. 


\section{AUTEURS}

\section{RÉMY PONGE}

Laboratoire Printemps (Professions, institutions, temporalités ; CNRS, UMR 8085), université de Versailles - Saint-Quentin-en-Yvelines 